\title{
Dos fragmentos de Heráclito acerca de psyche $y$ logos
}

\author{
Enrique Hülsz Piccone
}

uc» y | Ògoj son dos conceptos cardinales en la filosofía y la cultura griegas, (en particular en los siglos v y IV), que adquirieron su impulso más decisivo con Heráclito. Las páginas siguientes son una aproximación a los dos únicos fragmentos en que Heráclito vincula estos términos expresamente. ${ }^{1}$ El tema de su relación presenta derivaciones que este breve trabajo no pretende abarcar. Desde luego, ambas palabras son antiguas y sus sentidos diversos y cambiantes. Psyche, en Homero, aunque designa el principio vital en el hombre, sólo se aplica en forma negativa cuando el hombre viviente muere, o cuando ya ha muerto. El "ánima" o "alma" designa entonces su sombra o espectro en el Hades, cuando no es ya sino un triste reflejo degradado de la cabal existencia: la vida sobre la tierra. ${ }^{2}$ En el otro extremo histórico de la evolución de la palabra está quizá Platón, para quien el alma constituye lo esencial en el hombre, su ser más verdadero. Aunque seguro es cierto que el concepto de psyche representa el meollo del legado socrático, es probable que la idea de psyche haya adquirido el sentido de sede del pensamiento y la inteligencia ya en Heráclito, cuya tesis expresa e indudable es que hay un logos propio de psyche. Mi punto de

${ }^{1}$ Salvo pequeñas adiciones (las páginas introductorias acerca de logos), el cuerpo de este artículo forma parte de un trabajo más amplio, Logos. Un ensayo acerca de la filosofía de Heráclito. El presente abordaje supone una exploración previa del significado y la función del término logos en otros siete fragmentos donde recurre el término: B1: toà de I Ògou toàd' Òntoj a e ; ginom nwng r pentwnkat t n I Ògontònde; B2: toà I Ógoud' Òntoj xunoà;B31b:ka metr etai e j t naùt nI Ógon;B39:oá [sc. B...aj]

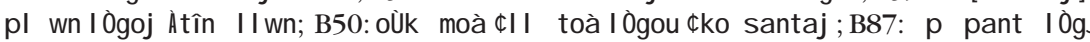
p toÁsqai;B108: kÓswn I Ògouj kousa o de j ktl. Respectode B72, asumo que la cita textual que puede atribuirse con justicia a Heráclito no incluye la palabra l Ogoj. Todas las referencias a los fragmentos se hacen según la numeración de Diels-Kranz.

${ }^{2}$ Cf. al respecto Nussbaum, M., "Y U C H in Heraclitus", en Phronesis, XVII (1972), núms. 1 y 2, pp. 1-16 y 153-170; Fränkel, H., Poesía y filosofía de la Grecia arcaica. Madrid, Visor, 1993, en especial, II, 6. 
partida aquí, cuya justificación sólo puedo esbozar brevemente, es la convicción de que logos es una noción compleja en los textos de Heráclito. Paradigma del concepto filosófico (a la vez categoría descriptiva ontológicognoseológica y fundamento moral prescriptivo), logos es también fórmula proporcional, metáfora e imagen. Todo esto confiere a la palabra una considerable densidad semántica, de modo que, con frecuencia, un mismo uso encubre más de un significado, y evoca resonancias de otros conceptos, otros nombres, otras imágenes. Por su parte, la versatilidad y el laconismo estilísticos de cada uno de los fragmentos, aunque dificultan la tarea interpretativa, son también, por cierto, lo que la motiva. La consideración atenta del estilo constituye una base indispensable en que ha de apoyarse toda lectura y toda interpretación globales. Las formas singulares de expresión -que van desde la narración hasta el aforismo y la sentencia gnómica, hasta el uso magistral de la paradoja, y el enigma- operan como un espejo del "sistema" entero, y muestran un mismo esquema recurrente: el ensamble de contrarios.

Visto como concepto de contenido, logos entraña en Heráclito una relación, abismal y al mismo tiempo de profunda intimidad, entre el ámbito del lenguaje (concentrado simbólicamente en la palabra como nombre de la cosa) y el de la cosa misma, es decir, la realidad significada por o con el nombre. El inicio del escrito (B1) dice:

De este logos real y verdadero siempre los hombres se tornan incapaces de comprensión, antes y después de haberlo escuchado por primera vez. Pues aunque todas las cosas suceden según este logos, aquellos parecen inexpertos experimentando palabras y hechos como los que yo expongo, dividiendo cada cosa según la naturaleza y mostrándola tal como es. A los demás hombres, en cambio, les pasa inadvertido cuanto hacen despiertos como cuanto hacen dormidos.

El sentido que logos tiene aquí, desde luego, implica las acepciones de "palabra", "discurso" y "lenguaje", puesto que los hombres lo escuchan, aunque sin duda no se reduce a ellas. Contra las interpretaciones reductivistas -las que sostienen que logos es el propio discurso de Heráclito y nada máspuede aducirse el argumento de que incluso los usos preheraclitanos de logos abarcan muy diversos matices de lo que llamamos "racionalidad", y no puede decirse con validez que su significado deba limitarse sólo a la dimensión lingüística. ${ }^{3}$ Traducir logos por el "mensaje", la "palabra", el libro y el "discurso"

${ }^{3} C f$. sobre este punto, por ejemplo, Michel Fattal, "Le logos d'Héraclite: un essai de traduction”, en Révue des Études Grecques, vol. xCIX, núm. 470-471, enero-junio de 1986, pp. $142-152$. 
o la "doctrina" de Heráclito implica empobrecer y deformar su sentido originario. Pues si bien logos es todo esto, es todavía algo más, y por tanto es otra cosa. La columna vertebral del proemio (B1) es el contraste de la permanente realidad-de-verdad de "este logos", según el cual "ocurren todas las cosas" y la empecinada incomprensión de los hombres, quienes tienen trato cotidiano con él y lo escuchan, pero sin entenderlo jamás, estando siempre, respecto de él, como dormidos o alienados. El logos del proemio se yuxtapone al yo del filósofo sin confundirse con él: es su propio discurso, pero es algo más, asequible a todos y evidente antes de que el filósofo diga nada. Designa posiblemente lo que podríamos llamar la racionalidad, en un sentido lo bastante amplio como para dar cabida a la estructura objetiva de lo real y al objeto y la naturaleza propios del saber. De ahí que pueda pensarse que logos también quiere decir "razón" (como causa, fundamento o motivo inherentes a la cosa misma, al menos en B1, en B2 y B50). Resulta importante, así, su aparente afinidad con physis, que anticipa el "orden universal" (kosmos, $f$. B30), la "ley" (nomos, $c f$. B114) cósmica, y la "proporción" y "medida" (B31b) de la realidad toda. Lo que Heráclito llama logos tiene, por ende, un fuerte sentido "objetivo", en función del cual adquiere su dignidad epistemológica suprema (pues es precisamente por su objetividad o realidad que es visto como verdadero). Además del discurso objetivo de lo real al que hay que prestar oído, y el contenido de tal discurso, logos puede designar los discursos o explicaciones verbales en general (B87, B108), e incluso la "reputación", "fama" o "buen nombre" (B39). A pesar de lo que pudiera parecer a primera vista, esta variedad de acepciones implica una comunidad, cifrada en el sentido filosófico, ontológicamente fuerte o duro, de "razón-de-ser".

Es importante reconocer este sentido en el logos, además de la dimensión lingüística. La dimensión ontológica tiene alcance universal irrestricto, y contiene las nociones de orden y medida, regularidad y legalidad inmanentes en las cosas mismas. El contenido medular de logos se formula como unidad e identidad racionales de los contrarios: "Habiendo escuchando, no a mí, sino al logos, es sabio convenir que todas las cosas son uno" (B50). Logos es en Heráclito la voz del ser: el lenguaje de lo real y la manifestación de la realidad en el lenguaje. El concepto de una racionalidad dinámica y omnicomprensiva constituye el marco de referencia y el hilo conductor que sintetiza y unifica las diversas acepciones que presenta el término en los textos, y es también el fundamento básico de legitimidad para atribuir al tema del logos rango de teoría. $^{4}$

${ }^{4} C f$. a este respecto el planteamiento inicial de T. M. Robinson (resuelto negativamente por este autor) en "Esiste una dottrina del logos in Eraclito?", en Atti del Symposium Heracliteum 1981, L. Rossetti, ed., vol. I. Edizioni dell'Ateneo, p. 65: "In 
El primero de los textos es el célebre fragmento 45:

Si vas a los límites del alma, no los

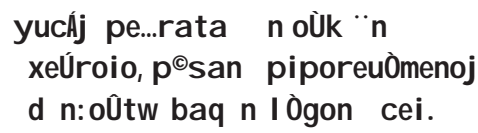

La forma negativa de la sentencia introduce, con cierta ambigüedad, una afirmación teórica. Lo intrincado de esta forma puede opacar la relativa claridad de la tesis central, que es la atribución de un logos a lo más hondo de psyche. En el contexto llaman la atención al menos tres cuestiones: los límites del alma; la imagen de una búsqueda interminable; y el sentido de la profundidad del logos.

A primera vista, parece natural aferrarse al texto explícito y entender que se concibe a psyche como limitada, ya que se le atribuyen expresamente unos confines o fronteras ( $p$ e...rata). Pero lo que se dice es que ninguna búsqueda llegará a esos límites. Lo cual resulta, en principio, paradójico: si psyche es finita, ¿por qué serían inalcanzables sus límites? De esta suerte, el tema de los límites se desliza hacia la cuestión de su inaccesibilidad, y B45 parece sugerir que psyche es "carente de límites, apeiron en un sentido más verdadero que "la inmensa tierra" ${ }^{6}$ En cualquier caso, el efecto inmediato de la cláusula "no encontrarías los límites del alma", es profundamente dialéctico: psyche aparece como limitada, a la vez que se sugiere su inmensidad. Más que establecer una prescripción moral, lo que se enuncia de modo categórico es la imposibilidad del hallazgo. La búsqueda, desde luego, queda así positivamente afirmada: sin ella, no tendría sentido hablar de límites inalcanzables. El cotejo con otros fragmentos realza el valor positivo que Heráclito parece atribuir a la búsqueda: "Quienes buscan oro cavan mucha tierra y encuentran poco" (B22); "Es necesario que [los varones filósofos] indaguen muchas cosas" (B35). Más revelador aún es B101, que consigna lo que, con dificultad, podría no ser una búsqueda interior: "Me busqué a mí mismo" ( dizhs fmhn mewut Òn). El punto central sería, así, la determinación de la búsqueda como un proceso de autoconocimiento inacabable.

breve, l'idea di una Logoslehre in Eraclito consiste nel supporre che logos per Eraclito significhi non solo il suo proprio discorso, ma anche la struttura organizzata della realtà descritta da tale logos".

${ }^{5}$ Agradezco al doctor Germán Viveros sus útiles sugerencias para la traducción de este fragmento.

${ }^{6}$ Charles H. Kahn, The art and thought of Heraclitus, p. 128 (ad B45). La referencia a los límites (inaccesibles) de la tierra es originariamente homérica. 
Por su parte, la alusión a psyche (que debe entenderse simultáneamente como "alma" y "vida") exige una elección interpretativa. Pudiera tomarse, por ejemplo, en sentido global e impersonal. Esta perspectiva genera una interpretación banal, para la que psyche no es mucho más que un tema o materia de discurso. A pesar de que las interpretaciones reductivistas de logos no resultan muy convincentes en otros casos, verter aquí logos por "discurso" no parece tan insensato: el discurso acerca de ella es tan profundo, que no se alcanzarían sus límites. Pero el eco de la dimensión lingüística parece, sin embargo, débil en el contexto de B45, además de que esta perspectiva vuelve al logos algo externo respecto de psyche (sería aquél, el discurso, el que contiene a ésta, no lo contrario). Resulta preferible comprender logos en B45 como "razón", en un ambiguo sentido donde confluyen "fundamento" y "conocimiento". Otro tipo de interpretación sitúa la búsqueda en un escenario cosmológico, y sostiene que la aplicación del término psyche no tendría restricción y abarcaría así la totalidad de los contenidos particulares del universo. Ésta es la perspectiva, por ejemplo, de Charles $\mathrm{H}$. Kahn, ${ }^{7}$ quien atribuye a Heráclito una concepción panpsiquista. La idea de esta lectura podría parafrasearse diciendo que el alma-vida no conoce fronteras, y que un viajero hipotético no llegaría nunca a su meta, si lo que buscara fuera la frontera o el confín de lo viviente. Pero esta visión parece leer en el fragmento mucho más de lo que éste dice: que "el alma está en todas las cosas" no es declaración explícita en éste ni en ningun otro fragmento, ni está necesariamente implicada.

De forma bastante natural, puede entenderse psyche como referida en especial a la condición humana, en toda su concreción. Aquí se habla, entonces, no del alma como principio cuasi cósmico, sino de cada psyche humana individual, y la fórmula "el logos de psyche" equivale a "el logos del hombre". Así, una traducción más certera sería: "A las fronteras de $t u$ alma no llegarás, aun recorriendo todos los caminos: tan profundo es el logos que posee".

\section{II}

Veamos, uno por uno, los principales puntos. En primer lugar, ¿qué son los límites del alma? La finitud que se predica del alma podría sugerir, por ejemplo, su mortalidad. ${ }^{8}$ La idea parece prometedora, y ciertamente B36 aporta la evidencia interna de que, para Heráclito, el alma es algo generado y mortal:

${ }^{7}$ Cf. íbidem, ad locorum (comentarios a B113 y B45).

${ }^{8}$ Así por ejemplo C. Eggers Lan (Los filósofos presocráticos, vol. I, núm. 90, p. 373): "En el caso presente [sc. B45] los 'límites del alma' son sin duda su nacimiento y su muerte. Pero su 'fundamento' o ‘sentido' $[=l o g o s]$ van más allá de ellos”. 
Para las almas muerte devenir agua, para el agua muerte devenir tierra; de la tierra nace el agua, del agua el alma. ${ }^{9}$

A pesar de la coincidencia aparente entre B45 y B36, a través de la idea de los límites, hay probablemente una lejanía contextual que desaconseja establecer en este caso un paralelismo demasiado simple entre ambos fragmentos. ${ }^{10}$ De cualquier modo, resulta en sí misma sugerente y atractiva la idea de que el alma muere. Notemos que, según B36, la muerte de las y u c a ... es un fenómeno tres veces dialéctico: primero, a) porque el alma, por definición, equivale a "principio vital", pero también b) porque "muerte del alma" es lo mismo que "génesis del agua", y c) porque implica diacrónicamente un [¿re-?]nacimiento del alma, que es muerte del agua. Así, el punto sobresaliente es la correlatividad simétrica de thanatos y genesis, muerte y nacimiento. El proceso descrito en $\mathrm{B} 36$ bien puede ser una ilustración del camino ascendente-descendente a que alude B60 ("El camino hacia arriba y hacia abajo [es] uno y el mismo"). ${ }^{11}$ En todo caso, la idea predominante en B36 es, sin duda, la de un equilibrio global que rige la vida y la muerte, en el que está implicado el logos.

Si trasponemos la mortalidad-generabilidad de B36 y la leemos en B45, los límites de psyche serían, pues, nacimiento (muerte del agua) y muerte (génesis del agua). El sentido del texto sería: "nacimiento y muerte del alma no podrías encontrar, aun recorriendo en tu marcha todos los caminos: tan profundo es su logos". La implicación parecería entonces ser una virtual identidad de logos y psyche, y la atribución a ésta de una condición eterna. Pero aproximar la búsqueda infructuosa (una imagen plausiblemente referida a la condición epistémica del hombre) al camino hacia arriba y hacia abajo

${ }^{9}$ B36: y ucí sin qfnatoj Ûdwr gen sqai, Ûdati d qfnatoj gán gen sqai: k gÁi d Ûdwr g...netai, x Ûdatoj deyuc.

${ }^{10}$ El contexto de aplicación de B36 no es necesariamente ontológico-cosmológico, sino que puede ser, por ejemplo, fisiológico, siendo agua y tierra sustitutos simbólicos de sangre, y carne y huesos, respectivamente. Vid., por ejemplo, Marcovich, Heraclitus. Greek text with a short commentary (editio maior). Mérida, Venezuela, 1967, pp. 361-364.

${ }^{11} \mathrm{~B} 60$, d j nw kftw m...a $k a \quad u t$ ». Este texto ofrece quizás una clave útil para interpretar B36: muerte es el camino "descendente" y génesis el "ascendente", dentro del balanceado cambio cualitativo cósmico -de cuyo esquema pudiera dudarse que sea, en sentido estricto, cíclico, aunque si se lo concibe como lineal, parece "reversible". De modo semejante, el punto en que $\mathrm{B} 31 \mathrm{~b}$ hace énfasis es la racionalidad del cambio cuando dice que la tierra, al devenir mar "es medida en la misma proporción (logos) en que existía antes". 
ilustrado en B36 parece un recurso demasiado forzado, por decir lo menos. Además, aunque la subordinación de los límites a la noción de logos como medida parecería prestarle consistencia a esa perspectiva, la inaccesibilidad de tales límites es algo que resulta bastante extraño y difícil de entender dentro del contexto específico. Y, finalmente, no se explica tampoco la frase final, o Ût w ba q n I Ògon cei, donde logos debiera tener el mismo sentido que exhibe en B31b (implícito en B36), "razón proporcional"; pero la noción de una proporción "profunda" es también escasamente inteligible ${ }^{12}$-lo cual impone deslizarse hacia la noción de logos como "medida", que resulta así un tanto reiterativa de la inaccesibilidad de los límites.

En este fragmento (B36), como ha sido notado con frecuencia, psyche ocupa un lugar protagónico en el proceso cíclico de transformación de "formas elementales" -y ofrece así el sentido de un principio o "elemento" cósmico, que tiene como pares a tierra y agua. Los límites que presumiblemente se definen ahí están en el horizonte del espacio y el tiempo cósmicos, e implican probablemente (a la luz de B31b) un sentido cuantitativo de racionalidad. En un contexto diverso (B85, un fragmento ético-fisiológico) reaparece esto último: "Difícil luchar contra el ánimo (thymos), pues lo que quiere lo paga con vida (psyche)". ${ }^{13}$ En general, se percibe con facilidad que el sentido de "vida" en la palabra y uc » es difícil de eliminar, y que está implicado con distintos grados de intensidad en las instancias heraclitanas. En B85, psyche representa el haber de vida de que se dispone, con el que se paga el precio de lo que thymos desea. Así, además de la noción genérica de "vida", lo único que parece conectar las formulaciones acerca de psyche en B45, B36 y B85 es la idea de limitación. Pero es manifiesto que ésta significa en cada caso cosas diferentes, y es, con seguridad, el contexto lo que determina la diversidad de las significaciones.

${ }^{12}$ Cf. A. García Calvo, Razón común. Edición crítica, ordenación, traducción y comentario de los restos del libro de Heráclito. Madrid, Lucina, 1985, ad loc., para una interpretación de logos en sentido cuantitativo.

${ }_{13}$ B85 provee una significación compatible con este sentido primario, donde psyche se opone a thymos: qumù mfcesqa i cal ep òn:0< $<\mathrm{i}>\mathrm{g} r$ " n q I V, y ucÁj ç ne tai: "Difícil luchar contra thymos ('ánimo', 'deseo', 'corazón'), pues lo que quiere, lo paga (o compra) con psyche ('alma', 'vida')". Psyche significa aquí "vida", y el contexto parece fisiológico (quizás con resonancias éticas). La finitud de psyche puede comprenderse como la magnitud limitada de la vida o la fuerza vital en el hombre, que se contrapone, en una relación de intercambio compensado, a lo que su thymos quiere o desea. Este texto, sin embargo, plantea numerosas cuestiones que involucran una prolija polémica de interpretación, respecto de la categoría de "fuego" en el "sistema", y también en torno del significado de qumòj , y reservamos su tratamiento para otro lugar. Un punto importante es que el obvio contraste de ánimo y ánima remite a la idea de un tercer término implícito, que es el dueño de ambos, y el cual, por tanto, no se reduce a ninguno. 


\section{III}

A pesar de que la conexión psyche-logos es innegablemente el punto central, conviene, pues, no perder de vista su dualidad. Según B45, no puede llegarse a los confines del alma por ningún camino, debido al logos profundo que ella tiene. En primer plano está la imagen de una marcha interminable, la cual se lleva a cabo siempre dentro de un inmenso territorio que no puede ser sino la psyche misma. Resulta así que B45 ilustra o ejemplifica la búsqueda de la propia mismidad, la práctica del autoconocimiento (marca distintiva del sentido vital del filosofar tanto en Heráclito como en Sócrates) de la que, como ya reconocimos antes, hay constancia en B101: "me he indagado a mí mismo". Quizá la frase inicial de B45, sin soslayar la finitud de psyche, no suponga ni aspire a una definición específica de sus límites, sino que apunte, entonces, a la idea de que el alma misma es, en algún sentido no especificado de antemano, ilimitada o inconmensurable. Atendiendo precisamente a la conjunción de psyche con ese logos que le pertenece, quizás no sobre insistir en que es obvio que también psyche tiene varios sentidos, y no se agota en el concepto de un principio cósmico - el cual, como hemos visto, no parece pertinente ni iluminador respecto de $\mathrm{B} 45$.

Ahora bien: en otro lugar, Heráclito concibe la psyche -de manera clara, pero también oblicua- como sede del lenguaje y de la acción de pensar, la acción reflexiva por excelencia: como aquello en el hombre que es capaz de logos (de oírlo y de expresarlo): "Malos testigos para los hombres los ojos y los oídos si tienen almas bárbaras". ${ }^{14}$ Queda bien claro que aquí se llama "bárbaras" a las psychai que no entienden el lenguaje de lo real, el logos, y que la implicación positiva es que, para un alma no bárbara, ojos y oídos son testigos fidedignos. Psyche puede así ser interpretada en B45, no tanto por analogía con el discurso cosmológico, como una "forma elemental" 15 equiparable a la tierra o al agua, sino, en los términos del propio texto, como sede del lenguaje-pensamiento racional y verdadero. Desde luego, al conjugar B107 con B45, la interpretación se vuelve, en cierto sentido, ontológicamente restrictiva: el alma con límites inalcanzables no sería el alma per se, ni cualquiera, sino sólo la que en B118 se califica como "seca y mejor". ${ }^{16}$ Verosímilmente, entonces, en concordancia con la imagen de la búsqueda interior, no se trate de los límites de su ser, sino de su poder, su dynamis cognitiva. Y en efecto parece un poder enorme ese que consiste

${ }^{14}$ B107: kako mfrturej chngrènoisin Ñf qal mo ka ta barbfrouj yuc j còntwn.

15 Tomo esta útil fórmula de la obra citada de Kahn.

${ }^{16}$ Sigo en este punto la sugerente interpretación de M. Conche, Héraclite. Fragments. París, PUF, pp. 358-360. 
en la posibilidad de conocerlo todo y decirlo todo. De este modo, el sentido de B45 no sería que el alma fuese en sí misma ilimitada, pero sí que, en condiciones especiales ( $y$, en forma paradójica, abiertas a todos), su poder cognoscitivo es inmenso, pues todas las cosas son, en virtud de la presencia universal del logos, en principio inteligibles. Ésta es una tesis gnoseológica fundamentalmente optimista, y sólo en apariencia es incompatible con la devastadora crítica del aislamiento epistémico de los más ( $\begin{aligned} & 0 \\ & \text { p } 0 \mid\end{aligned}$ B17), o genéricamente "los hombres" ( nqr w p oi, B1) o (en contraste con el yo del propio Heráclito) los "otros hombres" (to j d e I I ouj фnqrèpouj, B1). En verdad, si la interpretación que proponemos es válida, esto arroja alguna luz sobre el abismo que parece mediar entre la concepción de la mayoría de los hombres como $\Varangle$ xÚnet 0 i , "ininteligentes", sordos a la voz del ser, quienes "estando presentes están ausentes" (B34), por una parte, y afirmaciones como "el pensar es común a todos" (B113), o "todos los hombres participan del conocerse a sí mismos y del ser sabios" (B116), por otra.

$\mathrm{Y}$ aun si bathys logos es traducido por "medida insondable", no parece muy distante del logos de B1, ley eterna según la cual todas las cosas suceden. Quizás sea incluso factible ver aquí el germen de los primeros usos de logos como racionalidad "subjetiva" o facultad de pensamiento. ${ }^{17} \mathrm{~B}$ a qúj , "hondo" o "profundo", es un epíteto naturalmente apropiado para el logos, en tanto que éste es visto como inmanente en la psyche. La profundidad con que el logos "cósmico" cala el alma misma sería fundamento de la agudeza con que el pensamiento verdadero hace presa en lo real. Y cuando Heráclito declara haberse indagado a sí mismo, queda implicado que lo que encuentra en su interior es el mismo logos que rige todo el universo. Es importante insistir en que logos no parece designar nunca en los fragmentos (incluyendo B45) la facultad cognoscitiva como tal, a la que se refiere en otros casos con palabras como noos, "intelecto" y phren, "pensamiento" (B40, B104, B114).

\section{IV}

El segundo texto, B115, es menos célebre, y por razones adicionales, de más difícil interpretación.

Del alma es el logos que se aumenta a símismo. yucÁ sti IÒgoj aut $n$ a xwn

\footnotetext{
${ }^{17}$ Análogamente a lo que ocurre en B2: di de pesqai tù xunù, tout sti tù> koinù (xun j g $r$ koinòj ): toà IÒgou d' Òntoj xunoà zèousin o pollo j d...an contej frònhsin: "Por eso es necesario seguir a lo común. Pero aunque el logos es común, los más viven como si tuviesen una inteligencia privada”.
} 
La interpretación de B115 debe enfrentar la dificultad preliminar de la autenticidad. ${ }^{18}$ La primera razón de suspicacia es lo incierto de la atribución: Estobeo, ${ }^{19}$ un autor del siglo v después de Cristo que es, en este caso, la única fuente, lo asigna expresamente a Sócrates, lo cual no impidió a Hermann Diels admitirlo en su Herakleitos (1901), basándose en la conjunción psychelogos de $\mathrm{B} 45$, y haciendo referencia, entre otras, a las fórmulas фnq r èp ou yuc n chnrèpla xetai ("el alma del hombre crece en el hombre") y chngrèp ou y uc ce fúetai m cri qfnatou ("elalma del hombre crece siempre hasta la muerte"), en textos hipocráticos. ${ }^{20}$ Con seguridad este proceder no resulta del todo satisfactorio según los más exigentes cánones críticos e implica un cierto optimismo, quizá infundado, ciertamente arriesgado.

Hay también quienes hacen suya la causa contraria. Entre éstos, Miroslav Marcovich aduce contra la autenticidad, primero, la atribución a Sócrates por Estobeo; luego, la semejanza con ciertos textos tardíos, de Aristóteles, Plotino, Plutarco, entre otros; ${ }^{21}$ y además, la incompatibilidad de la noción de un logos que se aumenta a sí mismo con la concepción de "medida", que "parece implicar algo constante en la física de Heráclito: una 'medida que se aumenta a sí misma' no es plausible en Heráclito". ${ }^{22}$ En cuanto a los dos primeros puntos, se diría que lo de la incierta atribución no representa un obstáculo insalvable para la defensa de la autenticidad, puesto que es razonable admitir una confusión del copista en el punto preciso en que aparece el fragmento en los manuscritos (luego de siete citas consecutivas de Heráclito). Los que niegan la autenticidad se topan desde luego con la incómoda cuestión de la atribución a Sócrates. Por la otra parte, la sospechosa similitud en textos muy tardíos con la noción del alma como "número que se mueve a sí mismo" no constituye, por sí sola, prueba de la inautenticidad de B115. Del mismo modo, pero a la inversa, la recurrencia de la noción de una a xhs i j del alma en escritos [pseudo] hipocráticos y en [el pseudo] Epicarmo (DKB2), no es tampoco corroboración indudable ni evidencia decisiva a su favor. La postura más sensata sería quizás un moderado agnosticismo de raíz, que considera el texto hipotéticamente genuino.

El último argumento de Marcovich parece, en cambio, más sólido que los otros dos. ¿Es realmente logos incompatible con la idea de "crecer por sí

18 Entre quienes rechazan B115, se cuentan Bywater (quien lo omitió en su edición) y Marcovich (pp. 569-570). Otros autores adoptan una postura más cauta, sin reconocer necesariamente la autenticidad (por ejemplo, Kahn).

${ }^{19}$ Flor., I, 180a (post B114).

${ }^{20}$ De victu, I, 6, y Epid., vi, 5, 1, respectivamente.

${ }^{21}$ Por ejemplo, Aristóteles, De anima, A 2, 404b29; Plotino, vI, 5 [cf. III, 6, 1, 31; v, 1, 5, 9 y ss.]; Plutarco, De an. procr. 1012.

${ }^{22}$ Marcovich, op. cit., p. 569. 
mismo" o "acrecentarse a sí mismo"? El punto que resalta inicialmente es que el sentido que se dé a logos es un factor de peso en la discusión de la autenticidad o inautenticidad del fragmento. La objeción de Marcovich presupone como resuelta la cuestión en la identificación de logos con una noción reducida de "medida" y una confianza infundada en que el único sentido viable para logos es aquí el de "razón numérica" [(numerical) ratio], cosa al menos discutible. Pero, aparte de esto, y al margen de la discusión de la autenticidad, la tesis de la improbabilidad de la idea de una medida no constante plantea una dificultad no desdeñable para nuestra interpretación, sobre todo por la evidente afinidad de contenido que percibimos entre B115 y B45. Quizás una vía de salida a esta aparente aporía sea reconocer que también la idea de medida admite matices distintos. Si se presupone un contexto "físico" y una suerte de monismo, la tesis de Marcovich quizás resulta pertinente. Cosa que no ocurre necesariamente en un contexto diverso, antropológico o ético, o incluso ontológico. Ciertamente, "el sol no rebasará sus medidas" (B94), pero "es necesario apagar la desmesura (Ûbr i j ) más que un incendio" (B43). Sin pretender excluir en el sentido de logos, como un matiz posible, o una acepción subordinada, la noción de medida -que podemos admitir, junto con la idea genérica de "fundamento" en B45-, resulta demasiado apresurado trasponer, de manera automática, el logos-medida a B115, que podría suscitar otras asociaciones concernientes a lo específico de la condición humana, proclive a la Ûbr i j o desmesura.

En el otro extremo está la postura de Marcel Conche, que es una defensa de la autenticidad de B115 basada explícitamente en un paralelo con Píndaro, reforzado con una referencia interna a $\mathrm{B} 39$. Conche sugiere que el logos que se aumenta a sí mismo se contrapone al logos que la divinidad (y el canto de los poetas) "hace crecer" (a $x$ ei $)^{23}$. Esta perspectiva requiere, a juicio de ese autor, entender logos como "discurso" (la piedra de toque de la interpretación de Conche, al menos nominalmente reductivista) y tiene a su favor el atractivo de llevar al extremo el reconocimiento de que el logos propio del alma es como un ser viviente, que en forma análoga al cuerpo, es capaz de crecimiento. La noción de una a xhs ij tÁj y ucÁj es quizá la consecuencia filosófica esencial, pero aunque pueda estar necesariamente implicada, sin duda lo que el texto dice es que es el logos del alma el que se

${ }^{23}$ Cf. M. Conche, op. cit., pp. 354-356. Los desarrollos de Conche prolongan el enfoque de Clémence Ramnoux, Héraclite ou l'homme entre les choses et les mots. París, Les Belles Lettres, 1968, pp. 116-126. Esta autora establece y explora la posible conexión con Empédocles B110 con mayor detalle ahí mismo, en especial en las pp. 166 yss. 
aumenta a sí mismo. ${ }^{24} \mathrm{Si} \mathrm{la} \mathrm{autenticidad} \mathrm{quedara} \mathrm{demostrada} \mathrm{-algo} \mathrm{que,} \mathrm{como}$ se ve, es difícil esperar-, con seguridad vendría a reforzar nuestra interpretación de B45: aunque el alma sería, en fecto, limitada, sus límites serían inalcanzables porque no serían fijos. ¿Cómo podrían serlo, si del logos profundo que la habita se dice que se acrecienta a sí mismo?

Concluyamos tentativamente que todo esto apunta a la idea de que el logos (principio de sentido del cosmos entero) adquiere, en la interioridad profunda del hombre, manifiesta en el habla y el pensamiento inteligentes, una dimensión de la que carece en el resto del orbe, y que es la frágil autoconciencia. ¿ $\mathrm{O}$ es desmesura pensar que no se trata tan sólo del incremento del hombre individual, que acrecienta su propio ser por medio de la adquisición de la sabiduría, sino del enriquecimiento y perfección del ser como tal, en su totalidad? Aunque esto podría parecer una idea anacrónica (y quizá pasaría por ser típicamente moderna), la unidad del logos (de donde deriva su reiterada comunidad) contiene y penetra el kosmos e incluye necesariamente la polis, dentro de la cual vive cada psyche. Mejor se diría, así pues, que el anacronismo radica en la escisión entre el universo mismo y esa parte suya, a través de la cual el logos habla de sí mismo.

${ }^{24}$ Cf. Eduardo Nicol, La primera teoría de la praxis. México, UNAM, 1978, p. 42: "En un fragmento que debiera ser famoso, el 115, aborda Heráclito esa interioridad del hombre para revelar que no es uniforme ni estable. El alma, nos dice, es susceptible de crecimiento. Este cambio, que es en verdad un crecimiento del hombre, paralelo al crecimiento corpóreo que trae la edad, se produce según una medida propia: y uc Á sti I Ògoj a ut $n$ a $x w n$. Todavía hoy nos parece asombroso el hallazgo de esa idea certera, aunque debiéramos estar familiarizados con ella por la metáfora del alma que 'se ensancha', empleada por la poesía". 